\title{
Association of VSNL1 with schizophrenia, frontal cortical function, and biological significance for its gene product as a modulator of cAMP levels and neuronal morphology
}

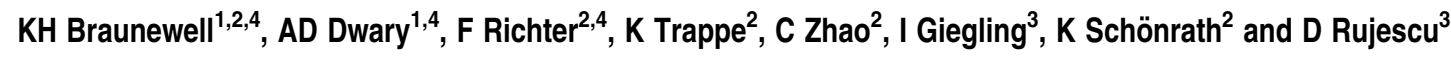

We report an association of single-nucleotide polymorphisms (SNPs) for the VSNL1 gene (visinin-like 1) with schizophrenia and frontal cortical function in a sample of patients with Diagnostic and Statistical Manual of Mental Disorder-IV (DSM-IV) diagnoses of schizophrenia, compared with healthy controls. Moreover, VSNL1 SNPs were associated with performance in the Wisconsin Card Sorting Test, a measure for the assessment of frontal cortical function. The VSNL1 gene product, Visinin-like-protein-1 (VILIP-1), is a member of the neuronal EF-hand $\mathrm{Ca}^{2+}$-sensor protein family. Previously, VILIP-1 mRNA and protein expression were shown to be altered in animal models and in schizophrenia patients. VILIP-1 influences cytosolic cyclic adenosine mono phosphate (cAMP) levels, cell migration, exocytotic processes and differentiation in the periphery. This raises the question, whether, similar to other potential schizophrenia susceptibility genes such as Disc1, PDE4B and Akt, VSNL1 may affect cAMP signaling and neurite outgrowth in neurons. In dissociated rat hippocampal neurons, VILIP-1 small interfering RNA knockdown decreased CAMP levels and reduced dendrite branching, compared with control-transfected cells. In contrast, VILIP-1 overexpression had the opposite effect. Similar results have been obtained in the human dopaminergic neuronal cell line SHSY5Y, where the effect on neurite branching and length was attenuated by the adenylyl cyclase inhibitor $2^{\prime}, 5^{\prime}$-dideoxyadenosine and the protein kinase A inhibitor KT5720. These results show that the association of VSNL1 SNPs with the disease and cognitive impairments, together with previously observed pathological changes in VILIP-1 protein expression, possibly occurring during brain development, may contribute to the morphological and functional deficits observed in schizophrenia.

Translational Psychiatry (2011) 1, e22; doi:10.1038/tp.2011.20; published online 19 July 2011

\section{Introduction}

Schizophrenia is a major disabilitating psychiatric disorder affecting approximately $1 \%$ of the population worldwide. The developmental disease originates from disturbances in very complex combination of genetic information, as well as environmental factors. ${ }^{1,2}$ About 2.2 million US citizens are diagnosed with schizophrenia, which, due to its early onset, results in an estimated cost for society of approximately $\$ 60$ billion annually. ${ }^{3}$ Pathological findings, including reduced interneuronal space and dendritic differentiation, decrease in dendritic markers, and a reduction in dendritic length, ${ }^{4-7}$ provide neuroanatomical evidence for the observed aberrant connectivity between brain structures, which may be the origin of psychotic symptoms and cognitive deficits. The consistent findings of ventricular enlargement and decreased brain volume in the hippocampus and cortex may be explained by decreases in dendrites and axons, a hypothesis known as the 'reduced neuropil hypothesis'. 4,8 Thus, it is likely that the underlying cellular mechanisms of the disease comprise developmental changes in the differentiation of dendritic and axonal structures of neurons. Indeed, growth factors, such as brain-derived neurotrophic factor and insulin-like growth factor-1 affecting neuronal differentiation, have a role in schizophrenia. ${ }^{9,10}$ Former identified putative susceptibility genes for schizophrenia, such as NRG1, Akt1, dysbindin-1 and $D I S C-1$ have all been implicated in neuronal differentiation and neurite formation in cellular and animal models of neurodevelopment. ${ }^{8,11-13}$ Disrupted in schizophrenia 1 (DISC1) is one of the most convincing genetic risk factors for major mental illness identified to date. DISC1 interacts directly with cyclic adenosine mono phosphate (cAMP)hydrolyzing enzyme phosphodiesterase $4 \mathrm{~B}$, an independently identified risk factor for schizophrenia. ${ }^{14}$ Moreover, the cAMP signaling pathway is implicated in shaping neuronal morphology during development. ${ }^{15}$

Different lines of evidence have accumulated indicating that neuronal calcium sensor proteins, such as visinin-like-protein-1

\footnotetext{
${ }^{1}$ Molecular and Cellular Neurosciences Laboratory, Department of Biochemistry and Molecular Biology, Southern Research Institute, Birmingham, AL, USA; ${ }^{2}$ Signal Transduction Research Group, Institute of Neurophysiology, Charité University Medicine Berlin, Berlin, Germany and ${ }^{3}$ Department of Psychiatry, Ludwig-MaximiliansUniversity, Munich, Germany

Correspondence: Dr KH Braunewell, Molecular and Cellular Neuroscience Laboratory, Department Biochemistry and Molecular Biology, Southern Research Institute, 2000 Ninth Avenue South, Birmingham, AL 35205, USA.

E-mail: braunewell@sri.org

${ }^{4}$ These authors contributed equally to this work.

Keywords: cyclic AMP; dendritic growth; dopaminergic; differentiation; hippocampal

Received 2 June 2011; accepted 3 June 2011
} 
(VILIP-1; gene name VSNL1 (visinin-like 1)) and neuronal calcium sensor-1, are associated with schizophrenia. Neuronal calcium sensor proteins are key players in different neuronal signaling pathways ${ }^{16,17}$ and are implicated in numerous pathological processes of central nervous system disorders. ${ }^{18}$ Recent genome-wide linkage studies in the Korean population have shown association of chromosome 2p24.3 with schizophrenia, where the VSNL1 gene lies. ${ }^{19}$ VSNL1 has also been correlated with cytoarchitectural abnormalities in schizophrenia and bipolar depression in the US population, with a differentiated gene expression found in the frontal cortex, ${ }^{20}$ identifying VSNL1 as a possible novel susceptibility factor. Earlier data from post-mortem studies in schizophrenic patients and in a ketamine schizophrenia model, revealed already reduced numbers and staining intensity of pyramidal neurons positive for the gene product VILIP-1. ${ }^{21-23}$ These results have been confirmed independently by microarray and proteomic studies. ${ }^{24,25}$ More recently, increased expression of microRNAs (for example, miR-181b) was discovered in schizophrenia and was found to negatively regulate VILIP-1 expression in schizophrenic brains and in human dopaminergic SH-SY5Y neurons, ${ }^{26}$ further adding to evidence implicating VILIP-1 in schizophrenia. Interestingly, insulin and IGF-1, which have also been associated with schizophrenia, increases the expression of genes that show pathological decrease in expression in schizophrenia, including VILIP-1 expression, in human SH-SY5Y model neurons. ${ }^{27}$

Originally, VSNL1, which is highly conserved between chick, mouse and human, was identified during a screen for developmentally upregulated genes in the chick visual cortex, where it shows enhanced expression levels during neuronal differentiation and axonal pathfinding. ${ }^{28}$ In vitro assays have shown that the protein upregulates cAMP levels and differentiation in various peripheral cells, such as pancreatic, kidney, skin and glioma cells. ${ }^{29-32}$ Interestingly, in mouse $\beta$-cells, overexpression of VILIP-1 increases cAMP, which is accompanied by increased cAMP-responsive element-binding protein gene expression. ${ }^{31}$ Moreover, in squamous cell carcinoma, VILIP-1 inhibits rhoA via elevating cAMP levels. ${ }^{32}$ The cAMP pathway and CAMP-responsive element-binding protein activation, as well as inhibition of the rhoA-ROCK pathway, have been implicated in promotion of neurite outgrowth and functional recovery of neurons. ${ }^{15,33}$ Thus, we speculate that VILIP-1, in conjunction with the cAMP pathway, has a role for neuronal differentiation.

The specific aims of the study were to evaluate whether VSNL1 single-nucleotide polymorphisms (SNPs) show association with schizophrenia, and especially associate with one of the most sensitive measures for assessing impaired frontal cortical function, the Wisconsin Card Sorting Test (WCST). ${ }^{34}$ We next asked what the functional impact of VSNL1 SNPS and pathological expression of VILIP1 might be for neurodevelopment. Therefore, we have investigated whether VILIP-1 can influence cAMP signaling in neurons and have started to decipher its effect on neuronal morphology.

\section{Materials and methods}

Materials. Recombinant nerve growth factor and siRNA for VILIP-1 and control small interfering RNA (siRNA) were obtained from Invitrogen (Carlsbad, CA, USA). Forskolin, DDA (2',5'-dideoxyadenosine), H89 and KT5720 were from Tocris Biosciences (Ellisville, MO, USA) or from Sigma (St Louis, MO, USA). SMI-312 was from Covance (Austin, TX, USA). Affinity purified antibody against VILIP-1 was described previously. ${ }^{35}$

Cell culture and transfection of hippocampal neurons. Hippocampal cultures were prepared from fetal wistar rat brains (E18) and cultured as previously described. $^{35}$ Cells were plated onto poly-D-lysin coated 12-well culture plates at a density of 120000 cells for transient transfection experiments and onto 24-well culture plates with glass coverslips at a density of 60000 cells for immunocytochemistry. After $24 \mathrm{~h}$ of plating, culture medium was exchanged for neurobasal medium/b27 medium (Invitrogen), and hippocampal neurons were transfected after 1 day in culture with complementary DNA for green fluorescent protein (GFP), VILIP-1-GFP ${ }^{35}$ or siRNA, using the lipofectamin transfection method (Invitrogen) and cultured for $48 \mathrm{~h}$.

Cell culture and transfection of SH-SY5Y cells. SH-SY5Y cells were from ATCC (ATCC Number: CRL-2266, Salt Lake City, UT, USA) and cultured according to the suppliers instructions. For neurite outgrowth assays, $9000 \mathrm{SH}-\mathrm{SY} 5 \mathrm{Y}$ cells were placed in each 24-well cell culture plate with media containing $10 \%$ fetal bovine serum for $24 \mathrm{~h}$, followed by neurobasal/B27 medium. After $4 \mathrm{~h}$ of incubation, different chemicals were added to study the specific pathways involved (nerve growth factor and KT5720, $100 \mathrm{~nm}$; DDA, $300 \mu \mathrm{M})$ for an additional $48 \mathrm{~h}$. The Amaxa nucleofector system (Lonza, Cologne, Germany) was used for transfection with GFP and GFP-VILIP-1, or siRNA knockdown experiments with siRNA for VILIP-1 and scrambled control siRNA. Transfection efficiency was measured by counting percentage of cells showing green fluorescence, using a confocal microscope (TCS SPE, Leica Microsystems, Buffalo Grove, IL, USA).

Cell morphology analysis. Photographs of hippocampal neurons and SH-SY5Y neurons were analyzed with the Image J software (version1.41, NIH, http://rsb.info.nih.gov/ij/ index.html) using the Sholl and neuron J plugin. For Shollanalysis, quantification of transfected hippocampal neurons was performed by counting the number of neurite intersections of concentric circles placed around the center of the cell soma in gradually increasing radii from 20 to $100 \mu \mathrm{m}$. For SH-SY5Y cells, number and length of neurites longer than the diameter of one cell were measured using the neuron J plugin.

Western blot analysis. Hippocampal neurons (60000) or SH-SY5Y cells (10000) were cultured in 12-well cell culture plates for western blot analysis. After $48 \mathrm{~h}$ of transfection, the cells were lysed, and western blot analysis using primary anti-VILIP-1 antibody or anti-actin antibody for control was performed as previously described. ${ }^{35}$ 
Cyclic AMP assay. The production of cyclic adenosine mono phosphate (cAMP) was measured using the Amersham enzyme immunoassay kits (EIA 225, Amersham, Uppsala, Sweden) or the Cisbio HTRF c-AMP kit (Cisbio, Bedford, MA, USA) for hippocampal neurons and SH-SY5Y neurons, respectively. Transfected cells (60000 hippocampal neurons, $30000 \mathrm{SH}-\mathrm{SY} 5 \mathrm{Y}$ neurons) were analyzed following the manufacturers' protocols.

Schizophrenic patients. A total of 627 individuals with schizophrenia were ascertained from the Munich area in Germany. Of them, $66 \%$ were of German descent (that is, both parents were German) and 34\% were other Caucasians from middle Europe. Case participants had a DSM-IV and International Classification of Disease-10 (ICD-10) diagnosis of schizophrenia with the following subtypes: paranoid $78 \%$, disorganized $15 \%$, catatonic $2 \%$ and undifferentiated $5 \%$. Detailed medical and psychiatric histories were collected, including the Structured Clinical Interview for DSM-IV (SCID), to evaluate lifetime axis I and II diagnoses. $^{36,37}$ All details of this group of schizophrenic patients have been published elsewhere. ${ }^{38}$ Four physicians and one psychologist rated the SCID interviews and all measurements were double rated by a senior researcher. Exclusion criteria included a history of head injury or neurological diseases. All case participants were outpatients or stable inpatients.

Healthy controls. A total of 541 unrelated volunteers of German descent (that is, both parents German) were randomly selected from the general population of Munich, Germany, and contacted by mail. To exclude subjects with central neurological diseases and psychotic disorders or subjects who had first-degree relatives with psychotic disorders, several screenings were conducted before the volunteers were enrolled in the study. First, subjects who responded were initially screened by phone for the absence of neuropsychiatric disorders. Second, detailed medical and psychiatric histories were assessed for both themselves and their first-degree relatives by using a semi-structured interview. Third, if no exclusion criteria were fulfilled, they were invited to a comprehensive interview including the SCID I and SCID $\|^{36,37}$ to validate the absence of any lifetime psychotic disorder. Additionally, the Family History Assessment Module ${ }^{39}$ was conducted to exclude psychotic disorders among their first-degree relatives. Furthermore, a neurological examination was conducted to exclude subjects with current central nervous system impairment. In the case that the volunteers were older than 60 years, the Mini-Mental Status Test $^{40}$ was performed to exclude subjects with possible cognitive impairment. Written informed consent was obtained from all subjects after a detailed and extensive description of the study, which was approved by the local ethics committee and carried out in accordance to the ethical standards laid down in the Declarations of Helsinki.

Wisconsin card sorting test. A subset of the patients and controls were further characterized. A total of 566 (353 healthy volunteers, 213 patients) subjects participated in the WCST (Computer Version 4), ${ }^{41}$ where cards have to be sorted against a set of four standard cards according to a certain unknown rule, such as color, shape or number of symbols on the cards. Sorting rules changed according to predefined criteria.

Genotyping. DNA extraction was done with the QIAamp Blood Maxi Kit (QIAamp DNA Blood Midi/Maxi Handbook, Firma Qiagen, Hilden, Germany, 2005). DNA concentration was adjusted using the PicoGreen quantitation reagent (Invitrogen, Karlsruhe, Germany). The samples were genotyped using the Illumina HumanHap300 BeadChip according to the manufacturer's specifications (Illumina, San Diego, CA, USA). All solutions and reagents are from Illumina and were used according to the manufacturer's specifications. Several quality checks for genome-wide studies, like check for duplicates, check for gender or check for batch clusters were performed (for details see Stefansson et al. ${ }^{42}$ ). 266.382 SNPs passed the quality control in 1168 individuals with the following filters: (i) individual call rate $>95 \%$, (ii) SNP call rate $>99 \%$, (iii) minor allele frequency $<0.01$, (iv) deviation from HardyWeinberg equilibrium in controls at $P<10 \mathrm{E}-06$. After removing eight individuals, no population stratification was seen. For the chromosomal region of interest in this paper, SNPs were selected between the positions (Chr 2: 17518961 and 17665665) covering eight SNPs. Only samples with a call rate greater than $98 \%$ were included in the analysis. The Hardy-Weinberg equilibrium was $P>0.05$ in all SNPs and subsamples.

Statistical analysis. Significance of the neurite outgrowth results was analyzed using Microsoft excel and Insat software. Average neurite length was expressed as mean \pm s.e. One-way analysis of variance was used to evaluate significant differences between treatment conditions. Sociodemographic parameters such as $\chi^{2}-$, $t$-tests or analysis of variance were used as appropriate. For case-control studies, an additive model in PLINK was used. ${ }^{43}$ For analyses of WCST, the covariates 'age', 'gender' and 'education', as well as the 'disease status' in the whole sample were included. Again, an additive model was calculated. Haploview 4.0 (http://www.broad.mit.edu/mpg/ haploview/) was used to generate a linkage disequilibrium map ( $r 2$ and D', see Supplementary Figure 1). Tests for associations using multimarker haplotypes were performed using the software environment ' $R$ ' (http://www.Rproject.org). Global and single haplotype significance and odds ratios were calculated. Nominally significant asymptotic $P$-values were confirmed by permuting the case-control status over 10000 replicates to adjust for multiple testing and observing the maximum test statistic in each case. To compare quantitative traits between the haplotypes, the $R$ software package 'haplo.score' was used, integrating the covariates sex, age, education and diagnosis as appropriate. All nominally significant $P$-values were confirmed by permuting over 10000 replicates and observing the maximum test statistic (simulated $P$-value; sim.p). 
Results

Association of VSNL1 SNPs with schizophrenia. Here we report for the first time, an association of SNPs in the VSNL1 locus (protein name VILIP-1) with schizophrenia. Two intergenic SNPs, rs394874 and rs424827, located on chromosome 2p24.3 (17518961, 17577281), 5' of the VSNL1 gene encoding $\mathrm{C} / \mathrm{T}$ and $\mathrm{A} / \mathrm{G}$ polymorphisms, and all other intragenic SNPs (rs650275, rs834504, rs1519477, rs12470654, rs1033297 and rs11677051; chromosomal location: 17623649, 17665665) showed significant association $\quad(P=0.0080, \quad P=0.0097, \quad P=0.0178$, $P=0.0190, \quad P=0.0210, \quad P=0.0054, \quad P=0.0384 \quad$ and $P=0.0053$, respectively, Table 1) with schizophrenia. Regarding linkage disequilibrium, one block of all eight SNPs was detected with high to moderate D' (Supplementary Figure 1). Haplotype analysis could further confirm the casecontrol results, showing one haploytpe with a higher frequency in schizophrenia patients (CGTCTAAG, frequency cases versus controls $=27.7$ versus $24.0 \%$, $\left.\chi^{2}=4.034, \quad P=0.0446\right)$ and the opposite protective haplotype less frequent in patients (TACTCGGA, frequency cases versus controls $=23.7$ versus $28.2 \%, \chi^{2}=6.155$, $P=0.0131$, Table 2). These data identify $V S N L 1$ as a novel putative schizophrenia susceptibility gene.

Association of VSNL1 SNPs with WCST in schizophrenia. The WCST measures higher executive, so-called frontal lobe functions, and perseverative errors have been shown to correlate positively with the occurrence of schizophrenia. ${ }^{34}$ For two of the intragenic SNPs, rs12470654 and rs11677051, a replicated association for the number of completed categories (controls/patients/ combined: rs11677051: $P=0.0029 / P=0.0321 / P=0.0003$; rs12470654: $P=0.0442 / P=0.0247 / P=0.0028$ ), as well as for the schizophrenia-specific perseverative error was found in the WCST (rs11677051: $P=0.0095 / P=0.0180 /$ $P=0.0004 ; \quad$ rs $12470654: \quad P=0.0401 / P=0.0141 /$ $P=0.0012$ ), with a lower performance in rs11677051 Acarriers and in rs12470654 in G-carriers. To avoid multiple testing problems, only associations, which replicated in both groups with the same risk allele (healthy controls and schizophrenia patients) as well as in the combined group, are presented here (for all data see Supplementary Material, Table 1). These results indicate that VSNL1 may be implicated in cognition and specifically in frontal cortexdependent cognitive impairments in schizophrenia.

Effect of modulation of VILIP-1 expression on CAMP signaling in hippocampal neurons. To look into the possible biological significance of the association of VSNL1 SNPs with the occurrence of schizophrenia, we evaluated the possible effect of pathologically altered expression of its gene product VILIP-1 on neuronal differentiation. We first examined localization of a heterologously expressed VILIP-1-GFP construct in hippocampal neurons. Fluorescence microscopy revealed localization throughout hippocampal neurons in soma and all processes (Figures $1 \mathrm{a}$ and $\mathrm{b}$ ). Accumulation in some hot spots along neurites and presumably neuritic growth cones is observed (Figure 1a, asterisks).

$\vdash \ll \vdash \cup \vdash \circlearrowleft \ll \varangle$

トலトレトல৫ல 
Costaining with the dendritic marker microtubule-associated protein 2 reveals that VILIP-1 is present in dendrites as well as in microtubule-associated protein 2-negative, axonal

Table 2 Association of VSNL1 SNP haplotypes with schizophrenia

\begin{tabular}{lccc}
\hline Block & $\begin{array}{c}\text { Frequencies (\%) } \\
\text { cases, controls }\end{array}$ & $\chi^{\mathbf{2} \text {-test }}$ & P-values \\
\hline CGCTCAGG & $0.327,0.311$ & 0.711 & 0.3991 \\
CGTCTAAG & $\mathbf{0 . 2 7 7}, \mathbf{0 . 2 4 0}$ & $\mathbf{4 . 0 3 4}$ & $\mathbf{0 . 0 4 4 6}$ \\
TACTCGGA & $\mathbf{0 . 2 3 7 , 0 . 2 8 2}$ & $\mathbf{6 . 1 5 5}$ & $\mathbf{0 . 0 1 3 1}$ \\
TACTCGGG & $0.086,0.088$ & 0.010 & 0.9206 \\
CGCTCGGA & $0.022,0.031$ & 2.114 & 0.1460 \\
TACTCAGG & $0.018,0.023$ & 0.670 & 0.4129 \\
CGTCTAGG & $0.018,0.013$ & 1.040 & 0.3079 \\
\hline
\end{tabular}

Abbreviations: SNP, single-nucleotide polymorphisms; VSNL1, visinin-like 1 Haplotypes of the eight studied SNPs and their $P$-values for case-contro association are presented. Haplotypes with a frequency $<1 \%$ were excluded. Significant haplotypes are in bold. extensions (Figure 1b), similar to our previous observations. ${ }^{35}$ To test whether VILIP-1-GFP expression may affect neuronal signaling and, in turn, neuronal differentiation of hippocampal neurons, we transfected E19 hippocampal neurons before differentiation in vitro with GFP-VILIP-1 and knocked down expression with VILIP-1 siRNA, and monitored expression by western blot analysis (Figures 1c and d). VILIP-1-GFP transfection produced an additional VILIP-1positive protein band at an molecular weight of $50 \mathrm{kDa}$ above the endogenous 22-kDa band (Figure 1c), whereas VILIP-1 siRNA knockdown reduced the expression of endogenous VILIP-1 by about $45 \%$ (Figures 1c and d). Next, the effect of VILIP-1-GFP overexpression or VILIP-1 knockdown on forskolin-stimulated adenylyl cyclase activity was evaluated (Figure 1e). A significant increase of cAMP levels following VILIP-1-GST transfection, and a decrease following siRNA knockdown of VILIP-1 expression indicated that VILIP-1 affects cAMP signaling in hippocampal neurons.
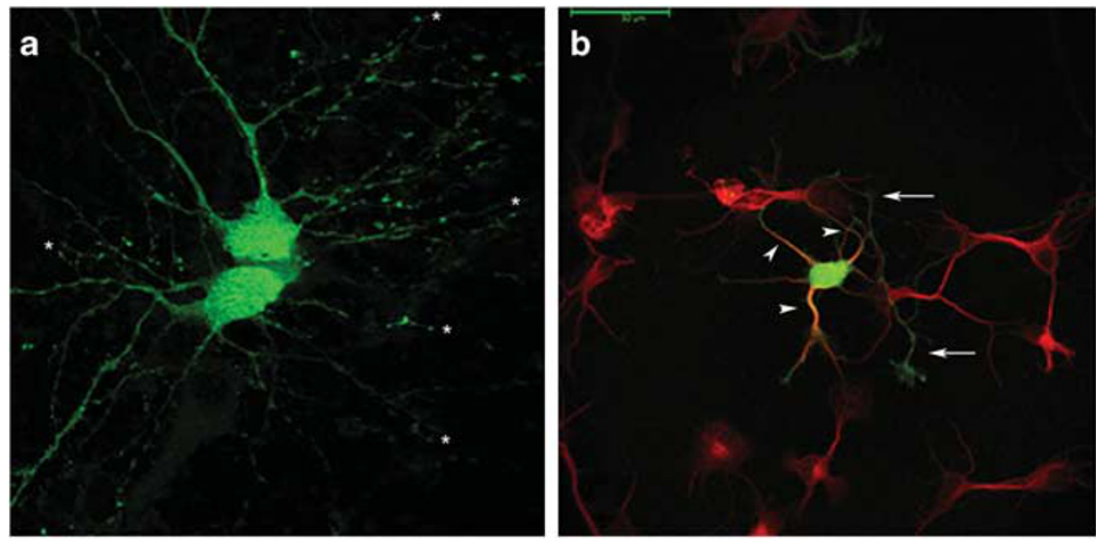

C
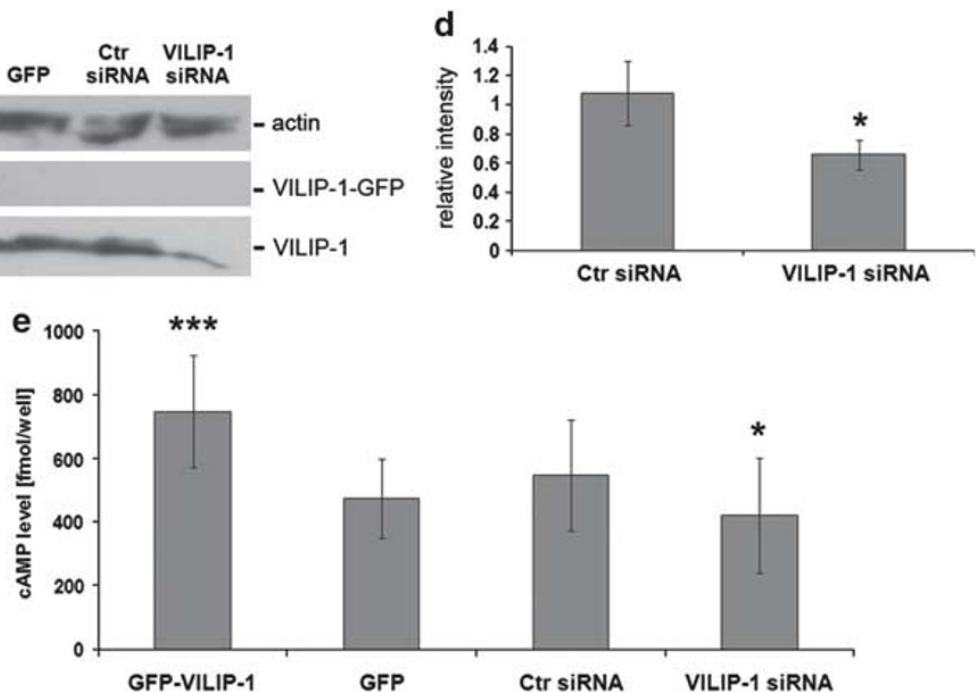

Figure 1 Visinin-like-protein-1 (VILIP-1)-green fluorescent protein (GFP) overexpression and small interfering RNA (siRNA) knockdown of endogenous VILIP-1 expression in hippocampal neurons. (a) Fluorescence detection of VILIP-1-GFP expression in two neighboring hippocampal neurons in culture. Note, hot spots of expression in fine neuritic extensions and growth cones (asterisks). (b) Localization of VILIP-1-GFP in axons and dendrites in VILIP-1-GFP-transfected (green) and non-transfected neurons costained with the dendritic marker microtubule-associated protein 2 (red). Note, co-staining in yellow is seen in dendritic structures (arrowheads), whereas only green fluorescence represents VILIP-1-GFP expression in axons (arrows). (c) Western blot analysis monitoring endogenous and VILIP-1-GFP overexpression (V1-GFP) and siRNA knockdown of endogenous VILIP-1 expression in hippocampal neurons. Immunostaining of actin serves as loading control. (d) Quantification of the effect of VILIP-1 siRNA knockdown (siRNA) of endogenous VILIP-1 expression. (e) Effect of VILIP-1-GFP overexpression (V1-GFP) compared with GFP control (empty), and of siRNA knockdown of endogenous VILIP-1 (siRNA) compared with scrambled siRNA control (scr) on forskolin-induced cAMP-accumulation in hippocampal neurons. Mean values from at least three independent experiments $(n=3)$ are shown and error bars indicate s.d. Asterisks indicate significant $\left(^{*}\right)$ and highly significant $\left(^{* \star *}\right)$ differences of the mean values. 
Effect of modulation of VILIP-1 expression on morphological differentiation of hippocampal neurons. When hippocampal cultures transfected with VILIP-1-GFP and GFP vector alone were compared, obvious changes in neuronal morphology of transfected cells were observed $48 \mathrm{~h}$ following transfection (Figures $2 \mathrm{a}$ and b). Compared with GFP control transfection, VILIP-1GFP transfection led to a noticeable stronger differentiation with a higher degree of branching (Figure 2, compare A with B). For quantification purposes, we aimed to identify the transfected neurons only, as hippocampal neurons show relatively low transfection efficiences. Therefore, we cotransfected a GFP construct with siRNA against VILIP-1 or the control siRNA (Figures $2 c$ and $d$ ). Only in the case of GFP-co-transfected neurons containing VILIP-1 siRNA, the expression of endogenous VILIP-1 was diminished (Figure 2c) compared with GFP/control siRNA co-transfected neurons (Figure 2d), as revealed by co-immunostaining against VILIP-1 protein (in red). Quantification of transfected hippocampal neurons was performed by Sholl analysis, which is a simple and established tool to characterize the morphological characteristics of neurons. ${ }^{44}$ Fluorescence images from GFP, VILIP-1-GFP, GFP/VILIP-1 siRNA and GFP/control siRNA-co-transfected hippocampal neuronal cultures, we analyzed using the half-automatic Sholl-Plugin for NIH ImageJ software. The number of dendrite intersections of concentric circles around the center of the cell soma was counted in gradually increasing radii from 20 to $100 \mu \mathrm{m}$ (Figure 3a). Overexpression of VILIP-1-GFP leads to increased number of dendritic arborization close to

a

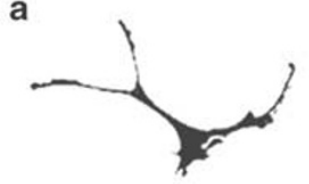

b
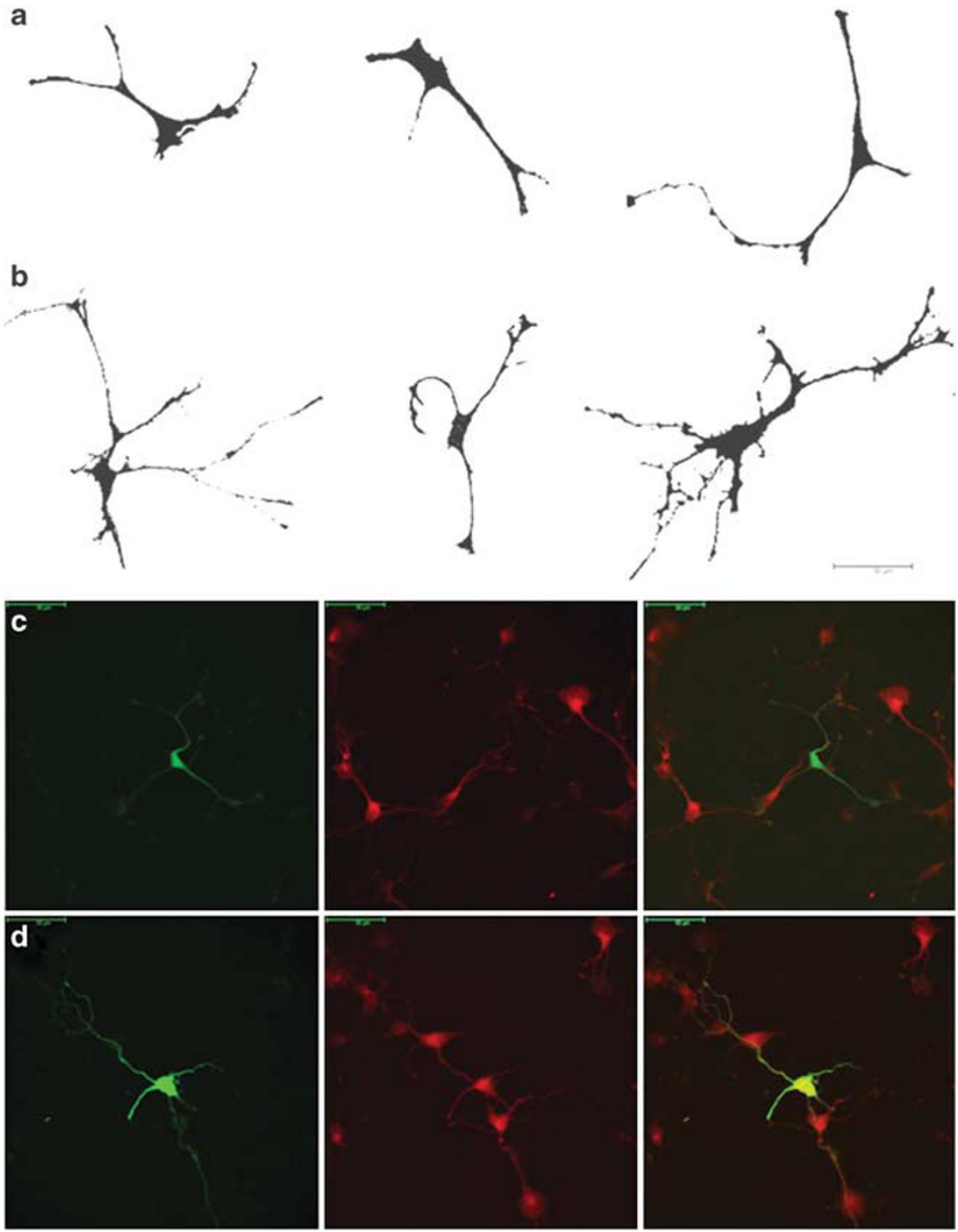

Figure 2 Effect of visinin-like-protein-1 (VILIP-1)-green fluorescent protein (GFP) on neuronal morphology and silencing of VILIP-1 expression with small interfering RNA (siRNA) in hippocampal cultures. (a and $\mathbf{b}$ ) Examples of dendritic morphologies of GFP (a) versus VILIP-1-GFP (b) transfected hippocampal neurons $48 \mathrm{~h}$ following transfection. Bar in B is $50 \mu \mathrm{m}$. (c and d) Silencing of VILIP-1 expression in hippocampal cultures with siRNA. Confocal fluorescence pictures GFP (green) and anti-VILIP-1- or control-siRNA co-transfected (after 1 div) hippocampal neurons (3 div) immunostained with anti-VILIP-1/Cy3 antibodies (red). (c) Shows anti-VILIP-1-siRNA-transfected, whereas (d) shows control-siRNA-transfected hippocampal neurons. The first column shows the green GFP channel, column 2 the red immunostaining channel for VILIP-1/ Cy3, and column 3 depicts the overlay of channel 1 and 2. Regions of colocalization of immunofluorescence appear yellow. Bars are $50 \mu \mathrm{m}$. 


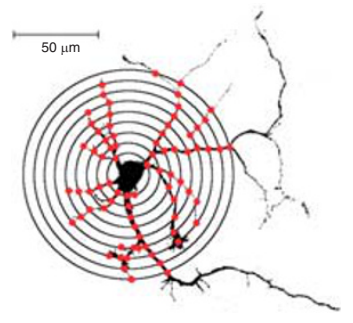

d

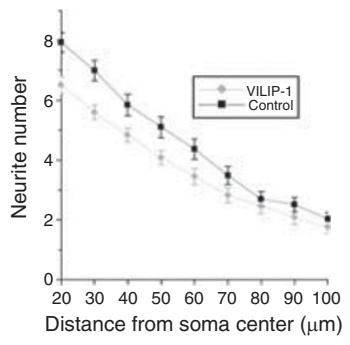

g.

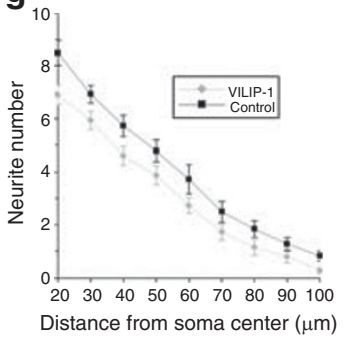

j

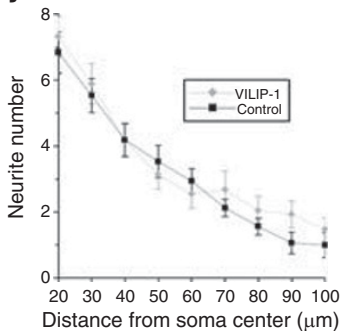

b

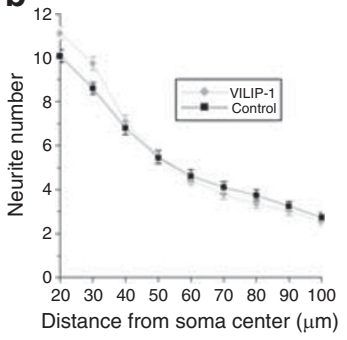

e

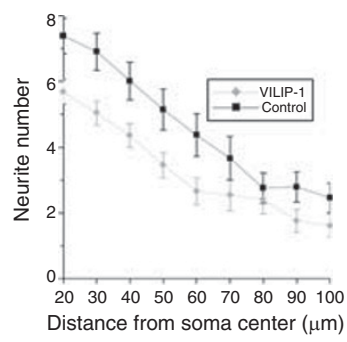

h

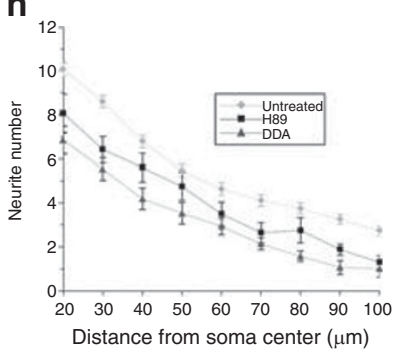

k

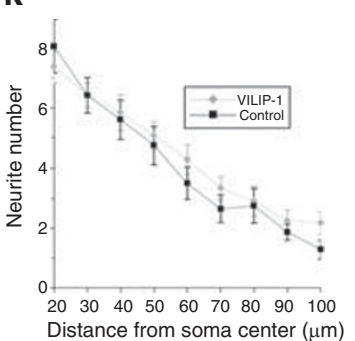

C

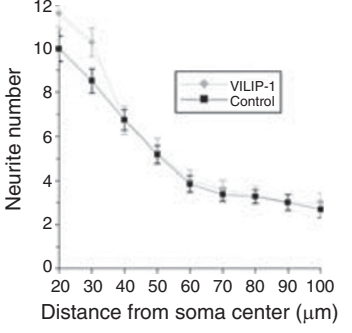

f

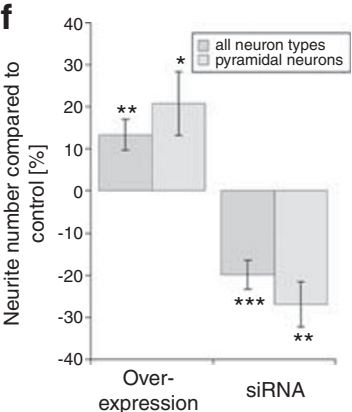

i
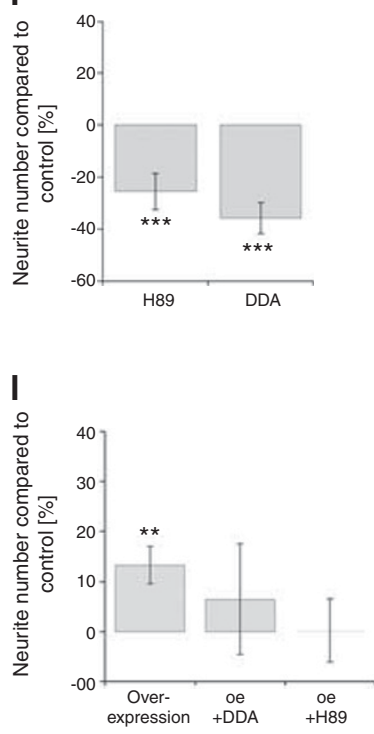

Figure 3 Effect of visinin-like-protein-1 (VILIP-1) expression and cyclic adenosine monophosphate (cAMP) signaling on branching in hippocampal neurons. (a) Exemplification of the Sholl analysis showing counting of neurites in concentric rings $(20-100 \mu \mathrm{m})$ around the cell soma of a hippocampal neuron as a measure for dendritic arborization. Bar in A is $50 \mu \mathrm{m}$. (b) Effect of VILIP-1-green fluorescent protein (GFP) overexpression (VILIP-1) on the number of dendrites in the distance from 20 to $100 \mu \mathrm{m}$ from the cell soma in all neurons. (c) Effect of VILIP-1-GFP overexpression on the number of dendrites in the distance from 20 to $100 \mu \mathrm{m}$ from the cell soma in pyramidal neurons. (d) Effect of VILIP-1-knockdown (VILIP-1) on the number of dendrites in the distance from 20 to $100 \mu \mathrm{m}$ from the cell soma in all neurons. (e) Effect of VILIP-1 knockdown on the number of dendrites in the distance from 20 to $100 \mu \mathrm{m}$ from the cell soma in pyramidal cells. (f) Summary of the effect of VILIP-1-GFP overexpression and VILIP-1 small interfering RNA (siRNA) knockdown on the number of dendrites in $30 \mu \mathrm{m}$ distance from the soma center in all neuron types and in pyramidal neurons. Data were from 100 neurons ( 25 for pyramidal neurons) per group out of three experiments. Asterisks indicate significant $\left({ }^{*}\right)$, high significant $\left({ }^{* *}\right)$, and highly significant $\left({ }^{* * *}\right)$ differences of the mean values. ( $\mathbf{g}-\mathbf{I})$ Effect of enhanced and reduced cAMP-signaling, and cAMP-dependent effect of VILIP-1 on branching in hippocampal neurons. ( $\mathbf{g}$ ) Effect of forskolin on the number of dendrites in the distance from 20 to $100 \mu \mathrm{m}$ from the cell soma in all neurons compared with control. (h) Effect of H89 and 2',5'-dideoxyadenosine (DDA) on the number of dendrites in the distance from 20 to $100 \mu \mathrm{m}$ from the cell soma compared with control. (i) Summary effect of DDA and H89 on the number of dendrites in the distance from 20 to $100 \mu \mathrm{m}$ from the cell soma in hippocampal neurons compared with control. (j) Effect of H89 on the VILIP-1-GFP-dependent increase in the number of dendrites in the distance from 20 to $100 \mu \mathrm{m}$ from the cell soma in all neurons. (k) Effect of DDA on VILIP-1-GFP-dependent increase in number of dendrites in the distance from 20 to $100 \mu \mathrm{m}$ from cell soma in all neurons. (I) Summary of the effect of DDA and H89 treatment on the VILIP-1-GFP-dependent increases in the number of dendrites in the distance from 20 to $100 \mu \mathrm{m}$ from the cell soma in all neurons. Data were from 100 neurons ( 25 for pyramidal neurons) per group out of three experiments.

the soma (Figures $3 b$ and $c)$. The greatest effect of VILIP-1 expression is observed at $30 \mu \mathrm{m}$ distance from the soma center when all neurons were analyzed (Figure $3 \mathrm{~b}$ ) and in neurons with typical pyramidal morphology (Figure 3c). When treatment with siRNA against VILIP-1 was compared with control siRNA treatment, significant differences in 
dendritic morphology was observed over a wider range of distance. VILIP-1 led to a significant decrease in the number of dendrites between 20 and $60 \mu \mathrm{m}$ distance from the cell soma in all neurons and in neurons with pyramidal phenotype (Figures $3 d$ and e). Quantification of the data at $30 \mu \mathrm{m}$ distance from the soma center showed a significant increase of $13 \%$ when all neurons were analyzed and a $21 \%$ increase in neurons with typical pyramidal morphology following VILIP-1-GFP transfection. siRNA for VILIP-1 produced $20 \%$ decrease in neurite number in all neurons versus $27 \%$ decrease for pyramidal cells (Figure 3f). These results indicate that VILIP-1 overexpression leads to formation of novel neurites close to the soma, whereas reduced VILIP-1 expression leads to a general reduction in the number of neurites at all distances from the soma.

Effect of VILIP-1 expression on morphological differentiation of hippocampal neurons depends on cAMP signaling. Next, we analyzed the causal correlation between the effect of VILIP-1 on CAMP production and its effect on dendritic arborization. As control treatment to show that cAMP affects neurite growth in the hippocampal culture system, forskolin was applied to enhance cAMP accumulation, and the general adenylyl cyclase inhibitor DDA and the protein kinase A inhibitor $\mathrm{H} 89$ were applied to reduce cAMP signaling in hippocampal neurons. As expected, these treatments led to enhancement and reduction of the number of dendrites, respectively (Figures $3 \mathrm{~g}$ and $\mathrm{h}$ ). Quantification of the effect revealed that the two inhibitors, H89 and DDA, significantly reduced neurite outgrowth by 25 and $35 \%$, respectively (Figure $3 i$ ). When VILIP-1-GFP-transfected hippocampal cultures were treated with the two inhibitors of the cAMP pathway, DDA and H89, the VILIP-1-dependent increase in number of dendrites was completely abolished (Figures 3j and k). Quantification of the effect revealed that the inhibitors significantly reduced the VILIP-1-dependent enhancement of dendritic growth to background levels (Figure 3I). Thus, the effect of VILIP-1 on formation of new dendritic processes depends on increased CAMP levels.

Overexpression and siRNA knockdown of VILIP-1 in SHSY5Y neurons and effect on cAMP signaling. The human neuronal cell line SH-SY5Y has been used as a cellular model for schizophrenia, in which growth factors implicated in schizophrenia lead to the regulation of schizophrenia susceptibility genes such as VSNL1. ${ }^{27}$ Therefore, we have tested the effect of VILIP-1 on neuronal morphology of SHSY5Y cells. VILIP-1-GFP expression in SH-SY5Y neurons was shown to be present in axonal as well as dendritic neurites, as revealed by co-staining with the axonal marker SMI-312 (Figures $4 a$ and b). Often, VILIP-1 was found to be enriched in growth cones of dendrites and axons (Figures $4 a$ and c, asterisks). The overexpression of VILIP-1-GFP in $\mathrm{SH}$ SY5Y neurons produced an additional VILIP-1-positive protein band at a molecular weight of $50 \mathrm{kDa}$ above the $22-\mathrm{kDa}$ endogenous protein band (Figure 4a), whereas VILIP-1 siRNA knockdown reduced the expression of endogenous VILIP-1 by $65 \%$ (Figures $4 \mathrm{a}$ and e). Moreover, VILIP-1-GFP overexpression has a significant effect on forskolin-stimulated cAMP accumulation, when compared with GFP-control-transfected neurons (Figure 4f). Significantly decreased cAMP levels were observed following siRNA knockdown of VILIP-1 expression (Figure 4g). These results indicate that VILIP-1 affects cAMP accumulation in the human neuronal cell line SH-SY5Y.

Effect of modulation of VILIP-1 expression and cAMP signaling on morphological differentiation of SH-SY5Y neurons. For evaluating the effect of VILIP-1 on dendritic growth, we transfected SH-SY-5Y cells with either GFP vector and VILIP-1-GFP, or with control siRNA and siRNA for VILIP-1, and quantified differences in number and average length of neurites, assuming that with only one axon per neuron, neurites will be mainly of dendritic origin (Figures 4a-c). VILIP-1-GFP overexpression (Figure 5, compare b with a) produces an increase, whereas siRNA treatment (Figure 5, compare d with c) leads to reduction in number and length of neurites as revealed by quantification of the average neurite length (Figure 5e). In addition, we used different chemicals, which are either activators or inhibitors of the cAMP signal pathway, to understand the exact mechanism by which VILIP-1 exerts its effects. The general inhibitor of adenylyl cyclases DDA and the protein kinase A inhibitor KT5720 were able to attenuate the VILIP-1 effect on neurite length in nerve growth factor-treated $\mathrm{SH}-\mathrm{SY} 5 \mathrm{Y}$ cells (Figure 5e). DDA reduced the average neurite length below control levels, which indicate that other factors besides VILIP-1 contribute to cAMP-dependent neurite extension in nerve growth factor-treated neurons. When additional parameters were analyzed, the number of branch points showed an even more pronounced effect. VILIP-1 increases dendrite branching by more than $100 \%$ (Figure $5 f$ ). Moreover, VILIP-1 seems to strongly induce formation of filopodia (Figure $5 \mathrm{~g}$ ). The examples show that in comparison with GFP-transfected SH-SY5Y cells, VILIP-1-GFPtransfected neurons show extensive branching, never observed in control cultures. Following quantification, a $100 \%$ increase in the number of neurons with extensive filopodia formation is observed (Figure $5 \mathrm{~h}$ ), indicating that VILIP-1 may particularly affect initial formation of filopodia, which typically proceeds neurite formation and dendritic branching.

\section{Discussion}

In this study, we have examined a set of SNPs located intragenic and intergenic $\left(5^{\prime}\right)$ of the $V S N L 1$ gene for association with the occurrence of schizophrenia in a cohort of patients and controls. ${ }^{38,42}$ We have identified significant association of two intergenic and six intragenic SNPs with schizophrenia, and two of the intragenic SNPs significantly associated with the WCST. ${ }^{41}$ For WCST, only replicated SNPs that were associated in cases only, in controls only and in the combined group with the same risk allele are highlighted. The WCST is used to assess higher cognitive functions, so-called executive functions, such as strategic planning, organized searching, utilizing environmental feedback to shift cognitive sets, directing behavior towards achieving a goal and modulating impulsive responding. ${ }^{34}$ 

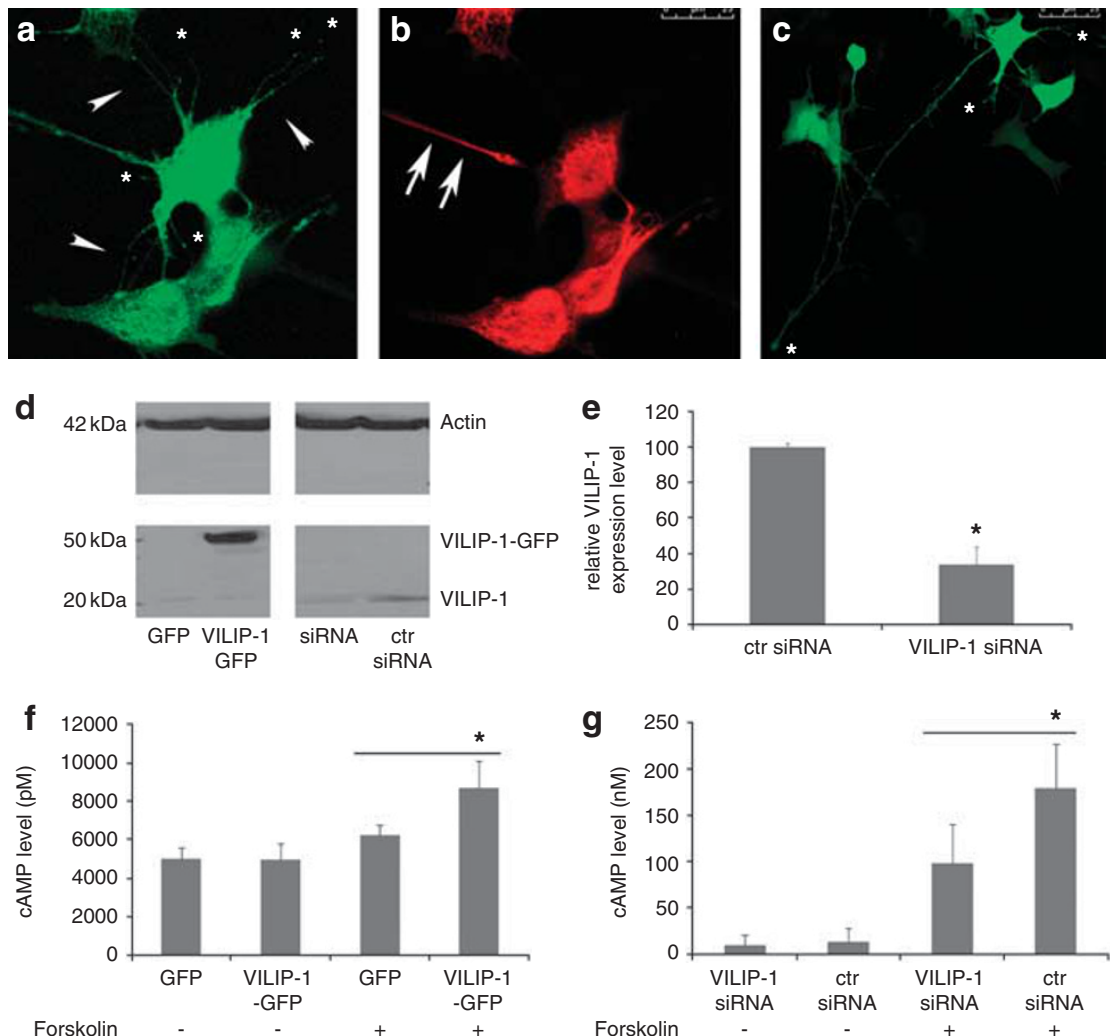

Figure 4 Visinin-like-protein-1 (VILIP-1)-green fluorescent protein (GFP) localization in human SH-SY5Y neurons and effect of VILIP-1-GFP overexpression and small interfering RNA (siRNA) knockdown of VILIP-1 on cyclic adenosine monophosphate (cAMP) accumulation. (a-c) Fluorescence picture of (a) VILIP-1-GFP transfected neuron, stained with (b) the axonal marker SMI-312. Arrow indicates a VILIP-1-GFP- and SMI-312-positive axon, whereas arrowheads indicate VILIP-1-positive, but SMI-312-negative dendrites. Asterisks show growth cones enriched with VILIP-1-GFP fluorescence. (c) Example of VILIP-1-GFP-transfected SH-SY5Y neuron with long, probably axonal structure and several dendritic-like neurites. Asterisks mark VILIP-1-GFP enriched growth cones. Bar is $25 \mu \mathrm{m}$. (d-g) Effect of VILIP-1-GFP overexpression and siRNA knockdown of VILIP-1 on cAMP accumulation in SH-SY5Y neurons. (d) Western blot analysis monitoring VILIP-1-GFP overexpression and siRNA knockdown of VILIP-1. Antibodies against VILIP-1 and against GFP stain endogenous VILIP-1 at $22 \mathrm{kDa}$ and a VILIP-1-GFP band at $45 \mathrm{kDa}$, respectively. Actin immunostaining serves a loading control. (e) Quantification of siRNA knockdown of VILIP-1 in SH-SY5Y neurons as shown in (d). (f) Effect of transfection of SH-SY5Y neurons with VILIP-1-GFP or GFP alone on basal and forskolin-induced cAMP levels. (g) Effect of transfection of SH-SY5Y with either VILIP-1 siRNA control or VILIP-1 siRNA on basal and forskolin-induced cAMP levels. Mean values from at least three independent experiments $(n=3)$ are shown and error bars indicate s.d. Asterisks indicate significant $\left(^{\star}\right)$ differences of the mean values.

The common test relies upon a number of intact cognitive functions including attention, working memory and visual processing. WCST perseverative errors have been shown to correlate positively with lesions of the dorsolateral frontal lobe and schizophrenia, suggesting that deficiencies in conceptual organization and flexibility in abstract thinking occur in patients. ${ }^{45}$ In this study, the relationship between the selected SNPs in the VSNL1 gene and cognitive set-shifting as measured by perseverative errors using the WCST was tested. The results indicate an association of cognitive flexibility and frontally based executive function with VSNL1 SNPS. However, it is noteworthy that counterintuitively the associated risk alleles for schizophrenia are associated with a better performance in the WCST. Although this finding may have been a surprise, similar results have also been presented; for example, for one of the best replicated risk genes for schizophrenia (ZNF804A) there, the schizophrenia allele was associated with better and not lower cognitive performance. ${ }^{46}$ Our results are therefore potentially important to our understanding of the genetic mechanisms and pathways that contribute to the susceptibility, as little is known about the function of VSNL1. The aim of our study was therefore to further characterize the functionality of this gene. Especially, as schizophrenia is a very heterogeneous disorder with several subtypes, it is perhaps possible that not all identified susceptibility alleles will have major effects on broad cognitive abilities and that, not all schizophrenia risk alleles will associate with all intermediate phenotypes in further functional studies.

Nevertheless, our results may hint towards an impact of VSNL1 SNPS and also of altered expression of VILIP-1 in schizophrenia on certain aspects of cognition. It is noteworthy in this context that VILIP-1 was identified as a cerebrospinal fluid biomarker for Alzheimer's disease with association of VILIP-1 levels, with the severity of Alzheimer's disease and association with the Mini-Mental Statement Examination as a measure of cognitive impairment in Alzheimer's disease. ${ }^{47,48}$ Future experiments will have to show whether VILIP-1 expression is linked to specific cognitive disturbances, such as attention and/or memory impairment.

Our findings further raise the question of the biological significance of misregulated expression of the novel schizo- 

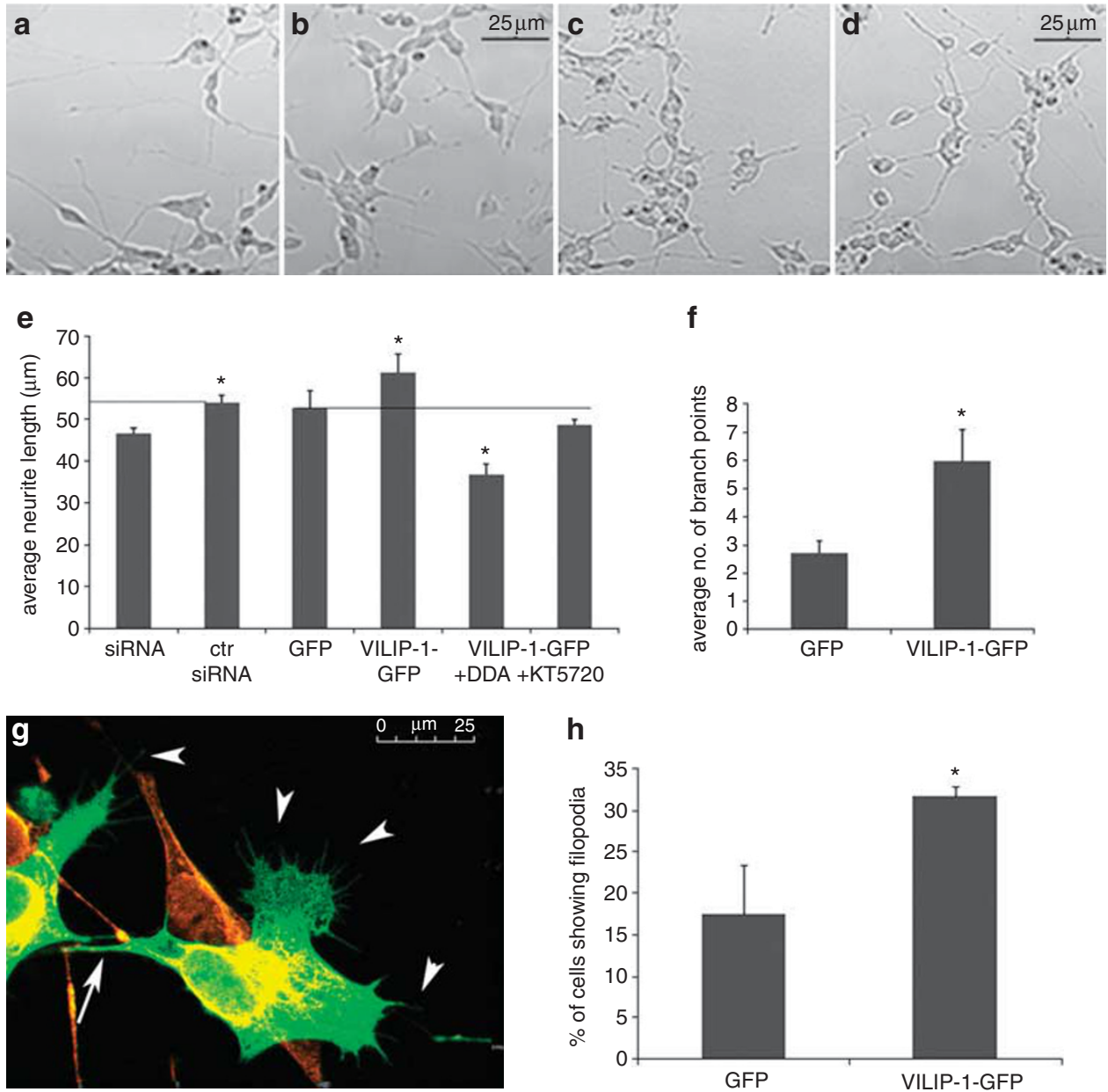

Figure 5 Effect of visinin-like-protein-1 (VILIP-1) expression and cyclic adenosine mono phosphate (CAMP) signaling on neurite outgrowth in nerve growth factor (NGF)treated human SH-SY5Y neurons. (a-c) Phase contrast images of SH-SY5Y neurons transfected with VILIP-1-green fluorescent protein (GFP) (a), GFP (b), VILIP-1 small interfering RNA (siRNA) (c) and siRNA control (d). (e) Quantification of the effect of VILIP-1- and CAMP signaling on average neurite length of NGF-treated human SH-SY5Y cells. Neurons were treated with VILIP-1 and control siRNA, transfected with GFP and VILIP-1-GFP, and transfected with VILIP-1-GFP and treated with adenylyl cyclase inhibitor 2',5'-dideoxyadenosine (DDA) or protein kinase A inhibitor KT5720. (f) Effect of VILIP-1-GFP transfection on average number of branch points in NGF-treated human SH-SY5Y neurons. Mean values from at least three independent experiments $(n=3)$ are shown and error bars indicate s.d. Asterisks indicate significant $\left({ }^{*}\right)$ differences of the mean values. Bars are $25 \mu \mathrm{m}$. (g) Example of a neuron extending high numbers of filopodia in NGF-treated VILIP-1-GFP-transfected human SH-SY5Y neurons. (h) Quantification of the number of neurons with filopodia in GFP- versus VILIP-1-GFP-transfected cells. Mean values were from at least three independent experiments $(n=3)$ and error bars indicate s.d.. Asterisks indicate significant $\left(^{*}\right)$ differences of the mean values. Bar is $25 \mu \mathrm{m}$.

phrenia susceptibility factor VILIP-1 during development, which may lead to structural and finally cognitive disturbances in affected brain regions. Therefore, we started to investigate whether upregulation and/or downregulation of VILIP-1 expression during development affect neuronal differentiation, and thus possibly lead to pathological changes in dendritic and axonal structures, which could contribute to developmental disturbances during the course of the disease. ${ }^{4,5}$ Interestingly, the schizophrenia susceptibility gene Disc 1 has recently been shown to have an effect on neuronal differentiation in that it enhances neurite outgrowth. ${ }^{14}$ Disc 1 is a physiological binding partner of PDE4, a cAMP-specific phosphodiesterase, thus potentially affecting the cAMP signaling pathway. ${ }^{15}$ Previously, VILIP-1 has been shown to affect cAMP-signaling in several heterologous expression systems. ${ }^{19,20}$ Reduced cAMP and increased rho-signaling lead to reduced branching of dendritic structures and are both important signaling system for neuronal differentiation. ${ }^{15,49}$ Thus, we have investigated the role of VILIP-1 in conjunction with cAMP-signaling for differentiation of hippocampal neurons and human SH-SY5Y neuronal cells, frequently serving as a cellular model for schizophrenia. ${ }^{26,27}$ We were able to show for the first time that VILIP-1 affects CAMP signaling in neuronal cells, and thereby affects cAMP-dependent morphology of neurons. Indeed, CAMP is a well-known promoter of neurite plasticity and branching. ${ }^{15,33,36,37}$ However, additional pathways regulating the fine tuning of axonal versus dendritic differentiation and outgrowth, such as GAP43, rhoA and the PIP2/PI3K/AKT/GSK3 $\beta$ pathway also have to be taken into account. ${ }^{49-53}$

One of the main characteristics of neurons is their shape, with every neuron extending long-branched dendrites to create a complex wiring network. Creation of such neuronal network has a central role in neuronal transmission and 
central nervous system function. VILIP-1-GFP led to enhanced branching, particularly in short distance from the soma in hippocampal neurons. Similarly, in SH-SY5Y neurons, VILIP-1 strongly induced formation of filopodia and branching. Both results indicate that VILIP-1 might affect already the initial phase of neuritogenesis and branching by enhancing the formation of new filopodia. During early development, filopodia can be transformed into dendritic branches, whereas later in development, filopodial protrusions can develop into dendritic spines. ${ }^{54,55}$ Thus, it appears likely that changed expression of VILIP-1 during development of the brain, depending on increased or decreased expression, may positively or negatively affect neuronal outgrowth and particularly, dendritic branching. The elaboration of complex dendritic arbors during development are necessary to integrate synaptic inputs and thus determine proper neuronal network activity. ${ }^{56}$ However, it also has been suggested that behavioral symptoms of schizophrenia could arise from over pruning or failed maintenance of dendrites later in life, occurring before and during psychosis development in schizophrenia in late adolescence. ${ }^{57}$ It is noteworthy in this context that the modulation of VILIP-1 expression has already been shown to affect neuronal network activity by affecting nicotinergic modulation of GABAergic activity of interneurons in hippocampal cultures. ${ }^{23}$ Observed pathological changes in expression levels, up- or downregulation in schizophrenia, for instance as reported in certain types of hippocampal interneurons and in the nucleus accumbens, ${ }^{22-26}$ might lead to pathological dendritic morphology of key neuronal subtypes. We hypothesize that this may contribute to subtle changes in neuronal connectivity, leading to some of the cognitive deficits typically measured via tests such as WCST.

\section{Conflict of interest}

The authors declare no conflict of interest.

Acknowledgements. This work was conducted within the framework of the national priority program (Schwerpunktprogram) SPP1226 Nicotine: Molecular and physiological mechanisms in the central nervous system (www.nicotineresearch.com) funded by the German Research Foundation (Deutsche Forschungsgemeinschaft, DFG, grants to KHB and DR), and with support from Southern Research Institute (www.southernresearch.org) to ADD and KHB.

1. Egan MF, Weinberger DR. Neurobiology of schizophrenia. Curr Opin Neurobiol 1997; 7: 701-707.

2. Lewis DA, Levitt P. Schizophrenia as a disorder of neurodevelopment. Annu Rev Neurosc 2002; 25: 409-432.

3. Saha S, Chant D, Welham J, McGrath J. A systematic review of the prevalence of schizophrenia. PLOS Med 2005; 2: e141.

4. Bellon A. New genes associated with schizophrenia in neurite formation: a review of cell culture experiments. Mol Psychiatry 2007; 12: 620-629.

5. Arnold SE, Lee VM-Y, Gur RE, Trojanowski JQ. Abnormal expression of two microtubuleassociated proteins (MAP2 and MAP5) in specific subfields of the hippocampal formation in schizophrenia. Proc Natl Acad Sci USA 1991; 88: 10850-10854.

6. Jones LB, Johnson N, Byne W. Alterations in MAP2 immunocytochemistry in area 9 and 32 of schizophrenic prefrontal cortex. Psychiatry Res 2002; 114: 137-148.

7. Villegas R, Villegas GM, Longart M, Hernandez M, Maqueira B, Buonanno A et al. Neuregulin found in cultured-sciatic nerve conditioned medium causes neuronal differentiation of PC12 cells. Brain Res 2000; 852: 305-318.

8. Selemon LD, Goldman-Rakic PS. The reduced neurophil hypothesis: a circuit based model of schizophrenia. Biol Psychiatry 1999; 45: 17-25.
9. Angelucci F, Brenè S, Mathé AA. BDNF in schizophrenia, depression and corresponding animal models. Mol Psychiatry 2005; 10: 345-352.

10. Venkatasubramanian G, Chittiprol S, Neelakantachar N, Naveen MN, Thirthall J, Gangadhar BN et al. Insulin and insulin-like growth factor-1 abnormalities in antipsychoticnaive schizophrenia. Am J Psychiatry 2007; 164: 1557-1560.

11. Laurino L, Wang XX, de la Houssaye BA, Sosa L, Dupraz S, Caceres A et al. PI3K activation by IGF-1 is essential for the regulation of membrane expansion at the nerve growth cone. J Cell Sci 2005; 118: 3653-3663.

12. Jiang $\mathrm{H}$, Guo W, Liang $\mathrm{X}$, Rao $\mathrm{Y}$. Both the establishment and the maintenance of neuronal polarity require active mechanisms: critical roles of GSK-3b and its upstream regulators. Cell 2005; 120: 123-135.

13. Miyoshi K, Honda A, Baba K, Taniguchi M, Oono K, Fujita T et al. Disrupted-InSchizophrenia 1, a candidate gene for schizophrenia, participates in neurite outgrowth. $\mathrm{Mol}$ Psychiatry 2003; 8: 685-694.

14. Millar JK, Pickard BS, Mackie S, James R, Christie S, Buchanan SR et al. DISC1 and $\mathrm{PDE} 4 \mathrm{~B}$ are interacting genetic factors in schizophrenia that regulate CAMP signaling. Science 2005; 310: 1187-1191.

15. Cai D, Qiu J, Cao Z, McAtee M, Bregman BS, Filbin MT. Neuronal cyclic AMP controls the developmental loss in ability of axons to regenerate. $J$ Neurosci 2001; 21: 4731-4739.

16. Braunewell $\mathrm{KH}$, Gundelfinger ED. Intracellular neuronal calcium sensor proteins, a family of EF-hand calcium binding proteins in search of a function. Cell Tissue Res 1999; 295: $1-12$

17. Burgoyne RD. Neuronal calcium sensor proteins: generating diversity in neuronal $\mathrm{Ca} 2+$ signalling. Nat Rev Neurosci 2007; 3: 182-193.

18. Braunewell K-H. The dark side of $\mathrm{Ca} 2+$ signaling by neuronal $\mathrm{Ca} 2+-$ sensor proteins: from Alzheimer's disease to cancer. Trends Pharmacol Sci 2005; 26: 345-351.

19. Hong KS, Won HH, Cho EY, Jeun HO, Cho SS, Lee YS et al. Genome-widely significant evidence of linkage of schizophrenia to chromosomes 2p24.3 and 6q27 in an SNP. Based analysis of Korean families. Am J Med Genet B Neuropsychiatr Genet 2009; 150B: 647-652.

20. Kim S, Webster MJ. Integrative genome-wide association analysis of cytoarchitectural abnormalities in the prefrontal cortex of psychiatric disorders. Mol Psychiatry 2011; 16: 452-461.

21. Bernstein HG, Braunewell KH, Spilker C, Danos P, Baumann B, Diekmann S et al. Hippocampal expression of the calcium sensor protein VILIP-1 in schizophrenia. Neuroreport 2002; 23: 393-396.

22. Bernstein HG, Becker A, Keilhoff G, Gorcyca WA, Braunewell KH, Grecksch G. Ketamine induced changes of the expression of neuronal calcium sensor proteins VILIP-1, -3 and hippocalcin in a partial schizophrenia model in rats. Neurosci Lett 2003; 339: 95-98.

23. Gierke $P$, Zhao $C$, Bernstein HG, Noack $C$, Anand R, Heinemann U et al. Implication of neuronal $\mathrm{Ca} 2+-$ sensor protein VILIP-1 in the glutamate hypothesis of schizophrenia. Neurobiol Dis 2008; 32: 162-175.

24. Miklos GL, Maleszka R. Microarray reality checks in the context of a complex disease. Nat Biotechnol 2004; 22: 615-621.

25. Vercauteren FGG, Flores G, Ma W, Chabot J-G, Geenen L, Clerens S et al. An organelle proteomic method to study neurotransmission-related proteins, applied to a neurodevelopmental model of schizophrenia. Proteomics 2007; 7: 3569-3579.

26. Beveridge NJ, Tooney PA, Carroll AP, Gardiner E, Bowden N, Scott RJ et al. Dysregulation of miRNA 181b in the temporal cortex in schizophrenia. Hum Mol Genet 2008; 17: $1156-1168$.

27. Altar CA, Hunt RA, Jurata LW, Webster MJ, Derby E, Gallagher P et al. Insulin, IGF-1, and muscarinic agonists modulate schizophrenia-associated genes in human neuroblastoma cells. Biol Psychiatry 2008; 64: 1077-1087.

28. Lenz SE, Henschel Y, Zopf D, Voss B, Gundelfinger ED. VILIP, a cognate protein of the retinal calcium binding proteins visinin and recoverin, is expressed in the developing chicken brain. Brain Res Mol Brain Res 1992; 151: 133-140.

29. Braunewell KH, Gundelfinger ED. Low level expression of calcium-sensor protein VILIP induces cAMP-dependent differentiation in rat C6 glioma cells. Neurosci Lett 1997; 234: 139-142.

30. Braunewell KH, Spilker C, Behnisch T, Gundelfinger ED. The neuronal calcium-sensor protein VILIP modulates cAMP accumulation in stably transfected $\mathrm{C} 6$ glioma cells: aminoterminal myristoylation determines functional activity. J Neurochem 1997; 68: 2129-2139.

31. Dai FF, Zhang Y, Kang Y, Wang Q, Gaisano HY, Braunewell KH et al. The neuronal Ca2+ sensor protein visinin-like protein-1 is expressed in pancreatic islets and regulates insulin secretion. J Biol Chem 2006; 281: 21942-22153.

32. Mahloogi H, González-Guerrico AM, Lopez De Cicco R, Bassi DE, Goodrow T et al. Overexpression of the calcium sensor visinin-like protein-1 leads to a cAMP-mediated decrease of in vivo and in vitro growth and invasiveness of squamous cell carcinoma cells. Cancer Res 2003; 63: 4997-5004.

33. Baptiste DC, Fehlings MG. Pharmacological approaches to repair the injured spinal cord. J Neurotrauma 2006; 23: 318-334.

34. Shad MU, Tamminga CA, Cullum M, Haas GL, Keshavan MS. Insight and frontal cortical function in schizophrenia: a review. Schizophr Res 2006; 86: 54-70.

35. Spilker C, Dresbach T, Braunewell KH. Reversible translocation and activity-dependent localization of the calcium-myristoyl switch protein VILIP-1 to different membrane compartments in living hippocampal neurons. J Neurosci 2002; 22: 7331-7339. 
36. First MB, Spitzer RL, Gibbon M, Williams BW, Benjamin L. Structured Clinical Interview for DSM-IV Axis II Personality Disorders (SCID-II). Biometrics Research Department, New York State Psychiatric Institute: New York, USA, 1990.

37. First MB, Spitzer RL, Gibbon M, Williams JB. Structured Clinical Interview for DSM-IV Axis I Disorders - Patient Edition (SCID - I/P, Version 2.0). Biometrics Research Department, New York State Psychiatric Institute: New York, USA, 1995.

38. Van den Oord EJ, Rujescu D, Robles JR, Giegling I, Birrell C, Bukszár J et al. Factor structure and external validity of the PANSS revisited. Schizophr Res 2006; 82: 213-223.

39. Rice JP, Reich T, Bucholz K, Neuman RJ, Fishman R, Rochberg N. Comparison of direct interview and family history diagnoses of alcohol dependence. Alcohol Clin Exp Res 1995; 19: 1018-1023.

40. Folstein MF, Folstein SE, McHugh PR. Mini-Mental State (a practical method for grading the state of patients for the clinician). J Psychiatr Res 1975; 12: 189-198.

41. Heaton RK. Wisconsin Card Sorting Test: Computer Version 4 - Research edition. Psychological Assessment Resources: Florida, USA, 2003.

42. Stefansson S, Ophoff RA, Steinberg S, Andreassen OA, Cichon S, Rujescu D et al. Common variants conferring risk of schizophrenia. Nature 2009; 460: 744-747.

43. Purcell S, Neale B, Todd-Brown K, Thomas L, Ferreira MAR, Bender D et al. PLINK: a toolset for whole-genome association and population-based linkage analysis. Am J Hum Genet 2007; 81: 559-575.

44. Sholl DA. Dendritic organization in the neurons of the visual and motor cortices of the cat. $J$ Anat 1953; 87: 387-406.

45. Prentice KJ, Gold JM, Buchanan RW. The Wisconsin Card Sorting impairment in schizophrenia is evident in the first four trials. Schizophr Res 2008; 106: 81-87.

46. Walters JT, Corvin A, Owen MJ, Williams H, Dragovic M, Quinn EM et al. Psychosis susceptibility gene ZNF804A and cognitive performance in schizophrenia. Arch Gen Psychiatry 2010; 67: 692-700.

47. Lee JM, Blennow K, Andreasen N, Laterza O, Modur V, Olander J et al. The brain injury biomarker VLP-1 is increased in the cerebrospinal fluid of Alzheimer disease patients. Clin Chem 2008; 54: 1617-1623.
48. Gómez Ravetti M, Rosso OA, Berretta R, Moscato P. Uncovering molecular biomarkers that correlate cognitive decline with the changes of hippocampus' gene expression profiles in Alzheimer's disease. PLoS One 2010; 15: e10153.

49. Hannila SS, Filbin MT. The role of cyclic AMP signaling in promoting axonal regeneration after spinal cord injury. Exp Neurol 2008; 209: 321-332.

50. Mingorance-Le Meur A, O'Connor TP. Neurite consolidation is an active process requiring constant repression of protrusive activity. EMBO J 2009; 28: 248-260.

51. Li H, Chen G, Zhou B, Duan S. Actin filament assembly by myristoylated alanine-rich $C$ kinase substrate-phosphatidylinositol-4,5-diphosphate signaling is critical for dendrite branching. Mol Biol Cell 2008; 19: 4804-4813.

52. Nguyen $\mathrm{L}, \mathrm{He} \mathrm{Q}$, Meiri KF. Regulation of GAP- 43 at serine 41 acts as a switch to modulate both intrinsic and extrinsic behaviors of growing neurons, via altered membrane distribution. Mol Cell Neurosci 2009; 41: 62-73.

53. Jan YN, Jan LY. Branching out: mechanisms of dendritic arborization. Nat Rev Neurosci 2010; 11: 316-328.

54. Dailey ME, Smith SJ. The dynamics of dendritic structure in developing hippocampal slices. J Neurosci 1996; 16: 2983-2994.

55. Yuste R, Bonhoeffer T. Genesis of dendritic spines: insights from ultrastructural and imaging studies. Nat Rev Neurosci 2004; 5: 24-34.

56. Ehlers MD. Dendrite development: a surprising origin. J Cell Biol 2005; 170: 517-519.

57. Karlsgodt KH, Sun D, Jimenez AM, Lutkenhoff ES, Willhite R, van Erp TG et al. Developmental disruptions in neural connectivity in the pathophysiology of schizophrenia. Dev Psychopathol 2008; 20: 1297-1327.

Translational Psychiatry is an open-access journal published by Nature Publishing Group. This work is licensed under the Creative Commons Attribution-Noncommercial-No Derivative Works 3.0 Unported License. To view a copy of this license, visit http://creativecommons.org/licenses/by-nc-nd/3.0/

Supplementary Information accompanies the paper on the Translational Psychiatry website (http://www.nature.com/tp) 